\title{
DESIGNING AND IMPLEMENTATION OF REMOTELY OPERATED COOKING MODULE
}

\author{
Rajesh Asutkar ${ }^{1}$, Gaurav Satav $^{2}$ \\ ${ }^{1}$ B.Tech, Electronics and Telecommunication, SGGSIE\&T, Nanded, Maharashtra, India \\ ${ }^{2}$ B.Tech, Electronics and Telecommunication, SGGSIE\&T, Nanded, Maharashtra, India
}

\begin{abstract}
In this paper report, efforts have been made in the direction of creating a machine that will enable a person to cook at multiple locations at the same time. This paper is motivated from the need that even after having raw material available ones disposal, the modern lifestyle prevents a person to eat healthy food due to lack of time in preparing it. The paper shows how to transform the recipe of a dish that one person will perform at a location into a digital format and send it over the wireless media to another location and then the same reverse action will be performed at the receiver location without having any human interference at the receiver in it. Cooking a recipe involves actions that are repeated over and over again and can be classified into a few basic operations; generally cooking involves heating a container in which we cook to a certain temperature, putting in the required ingredients after some time delay and stirring up the container to uniformly cook the food. Described module mainly consists of two nodes viz. active node where there is a necessity of a person to present to cook food. Passive Node is another one at which the food will be cooked automatically without requiring a person to be present there. The current paper will discuss the methods to digitalize the recipe, send the recipe data wirelessly to the remote location and recreate the same procedure as that of active node at the passive node.
\end{abstract}

Keywords: Active Node, Passive Node, Data Transmission, Data interpretation, Weight difference, Timing parameters, Arduino, HX 711 Module, RF Module

\section{INTRODUCTION}

Statement: To devise a mechanism to collect the data of ingredients used in cooking and quantity of corresponding ingredients and the process performed on them; send this data to the remote location and to recreate the same procedure at the remote location.

To implement such a mechanism we divide our task in three parts-active node, transmission and passive node. The active node will collect the data about weight of the ingredients and processes done on them. In transmission we transmit the data to the passive node. At passive node we decode the data and perform the same action as performed at the active node. Briefly saying that the passive node is a more complex structure as we have to devise a system similar to an automatic cooking machine but with the flexibility to perform the different process according to received data.

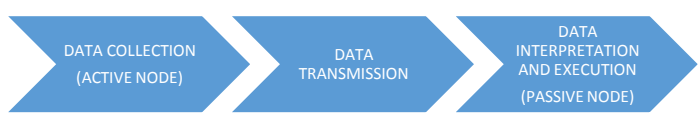

Fig -1: Block diagram of module

Precise idea: In this paper we try to collect the precise data about process performed at active node and transmit it and display it. Yet the scope remains for building up a passive node which enables the full functioning of the idea.
With a functioning passive node we will be able to cook the recipe which is being cooked at a distant place. As the data can be stored at passive node hence once performed process can be recreated at any wanted time. One can subscribe to a cooking show on television and will be able to cook the same recipe at the same time or later. The passive node provides the person with a comfort of not remembering the process while watching or writing it down and then cook. $\mathrm{He} / \mathrm{she}$ can do other activities while the recipe is being prepared as the human interference not required at passive node.

The following chapters contain detailed description about the active node, transmission and passive node. The hardware description is also included about the different devices used to achieve the required task.

\section{METHODS}

\subsection{Working of Active Node}

The Active Node is name given to the module in the paper at which a person's physical presence will be required as he will perform the action of cooking. At this node active participation of a human being will be required as he will cook the food. The sensor will continuously monitor the action of the person and will measure the appropriate parameters like the temperature of the container [6], weight of the ingredient used, the time interval between insertion of ingredients, etc. After getting this information the node will also perform the operation of sending this data wirelessly to 
the passive node after coding it. The wireless device that we have used is RF Module (433 MHz) [7]. In brief the complete working of the Active Node can be summarized as follows:

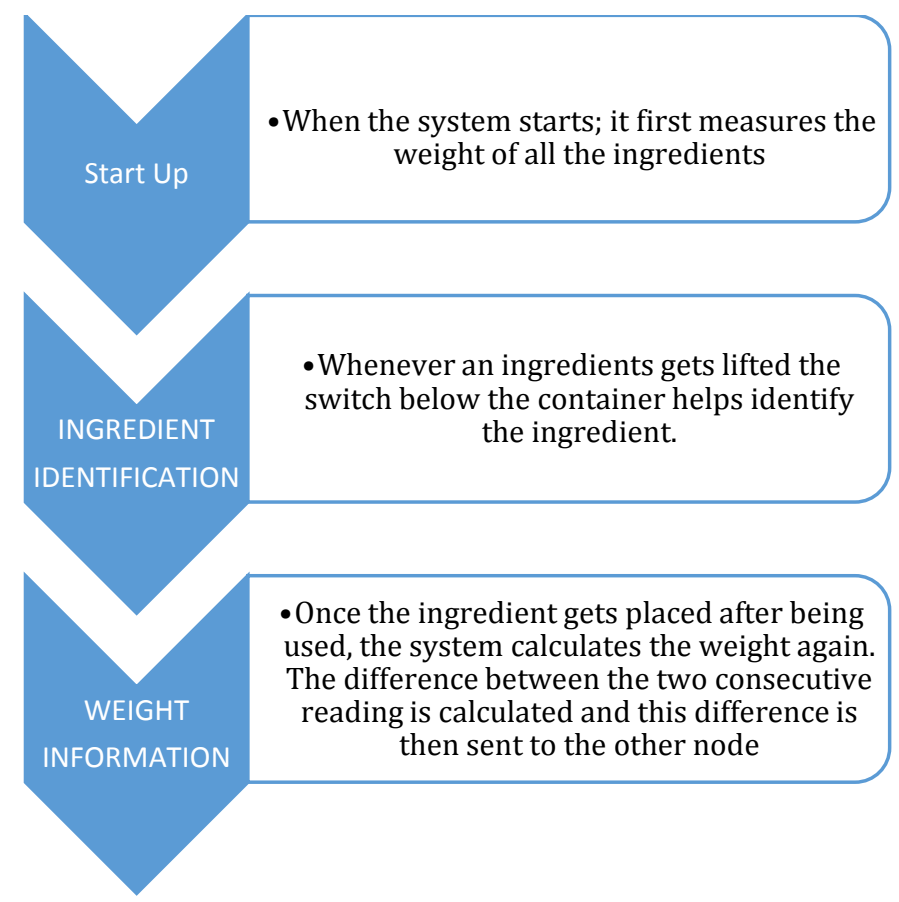

Fig -2: Working of an active node.

\section{List of Components}

$\begin{array}{ll}- & \text { Sensors. } \\ \text { - } & \text { RF Transmitter. } \\ \text { - } & \text { Microcontroller ATMega } 328 . \\ \text { - } & \text { Real Time Clock (RTC). } \\ \text { - } & \text { Induction Cooker. }\end{array}$

Table -1: List of sensors for active node

\begin{tabular}{|l|l|}
\hline List of Sensors & $\begin{array}{l}\text { Functional Description in } \\
\text { brief }\end{array}$ \\
\hline Limit Switches & $\begin{array}{l}\text { Used in identification of } \\
\text { ingredients. }\end{array}$ \\
\hline Load cell & $\begin{array}{l}\text { Used to measure the weight } \\
\text { of the ingredient used. }\end{array}$ \\
\hline $\begin{array}{l}\text { Resistor Temperature } \\
\text { Device (RTD). }\end{array}$ & $\begin{array}{l}\text { To measure the temperature } \\
\text { of the container. }\end{array}$ \\
\hline
\end{tabular}

The complete working of the Active Node will be explained with the help of an example. One very famous Indian recipe is the poha [4]. Let us see how the complete system will work. Initially we require the ingredients to have been place in the same order at both the nodes. For this the person at the Active Node must provide the person at the Passive Node with the information about the location of the ingredients. The ingredients that will be required are poha, groundnuts, oil, turmeric, salt, etc. The ingredients are then arranged in the order given above on the Active Node. Once all the ingredients are placed the system will note down the complete weight of all the ingredients. Let it be $x$ grams. The person will heat the container to a certain temperature $t$ degree Celsius. When a person will lift the first container containing poha, the switch underneath the container will trigger a local timer clock, since this is the first ingredient it will start with $O \mathrm{sec}$ and when he places it back after inserting some quantity of it in the cooking container, the system will identify which ingredient has been lifted with the help of the switch which is placed underneath all the ingredients containers. Also when the container is placed back the system will reweight all the ingredients and let the measurement of the weight taken this time be $y \mathrm{gm}$. The amount of ingredient that was used will then be,

$$
\text { Weight of ingredient used }(\mathrm{z})=(\mathrm{x}-\mathrm{y}) \mathrm{gm}
$$

After the insertion of the first ingredient let the second ingredient be used at time $t$, then the delay between the insertion will be given by

$\mathrm{T}=\mathrm{t}$ (time at which the second ingredient is lifted) -0 (time at which the first ingredient is lifted) seconds

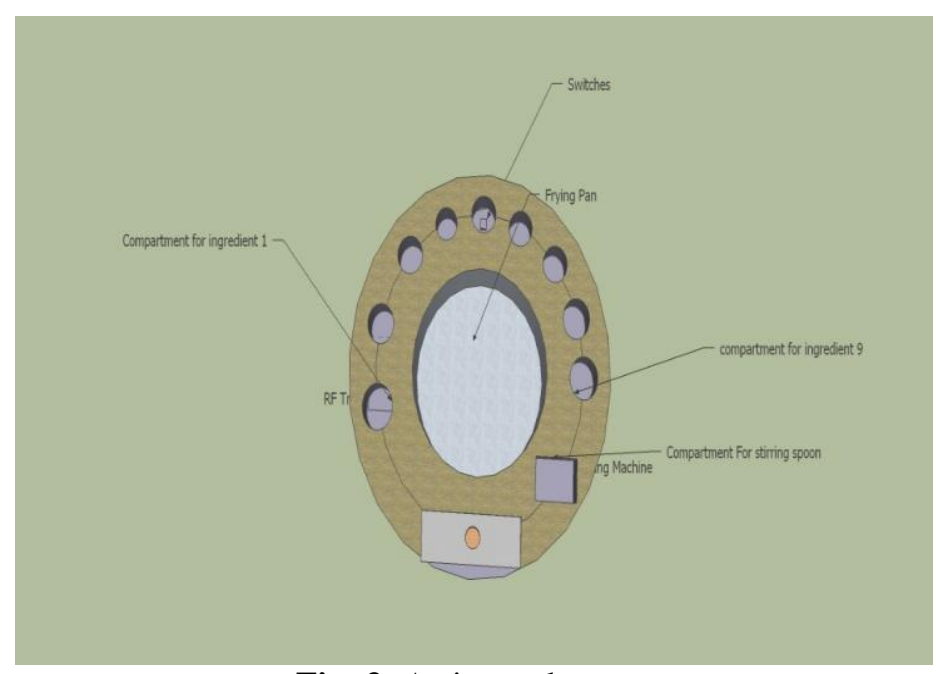

Fig -3: Active node

So now we have all the information necessary to send the detailed information about the first ingredient, which is

- Which ingredient has been used.

- What was the temperature of the cooking utensil when the first ingredient was inserted.

- What was the quantity of the used ingredient.

- After what time was the second ingredient used.

This data will be transmitted with the help of the RF Transmitter module to the Passive Node.

At the experimental level we have used limit switches for the identification of which ingredient has been pick up which is interfaced to Atmega 328 microcontroller on Arduino board [5]. For weighing the inserted ingredient we have used the load cell which has an aluminum bar in a cantilever arrangement and which is interfaced to Atmega 328 in bridge connection via Hx711 module [6]. We are using the onboard Real Time Clock (RTC) on the Arduino Board to measure the timing parameters 


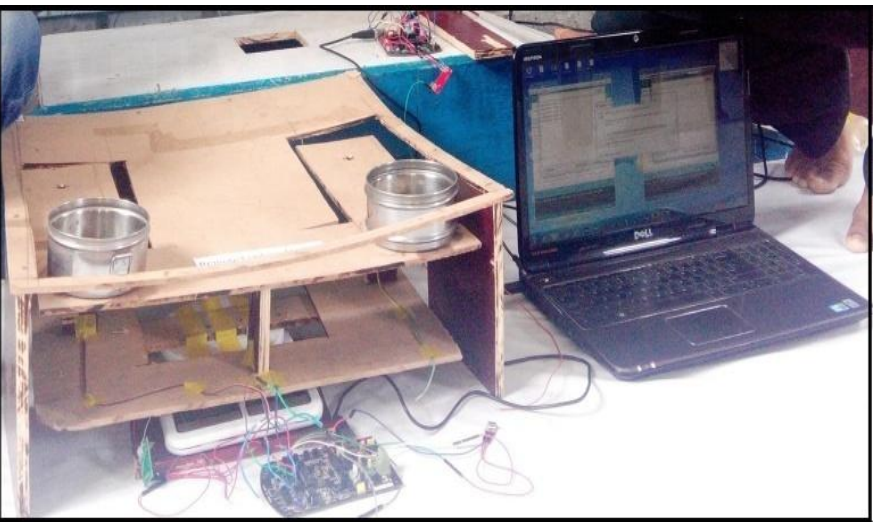

Fig -4: Experimental setup

\subsection{Working of Passive Node}

The Passive Node is the name given to the module at which the food will be cooked automatically without requiring a person to be present there. The Passive Node will basically perform the action of decoding the incoming data and carrying out the necessary actions. At this node we have used the RF Receiver which receives the data serially. The main actions performed by the Passive Node are

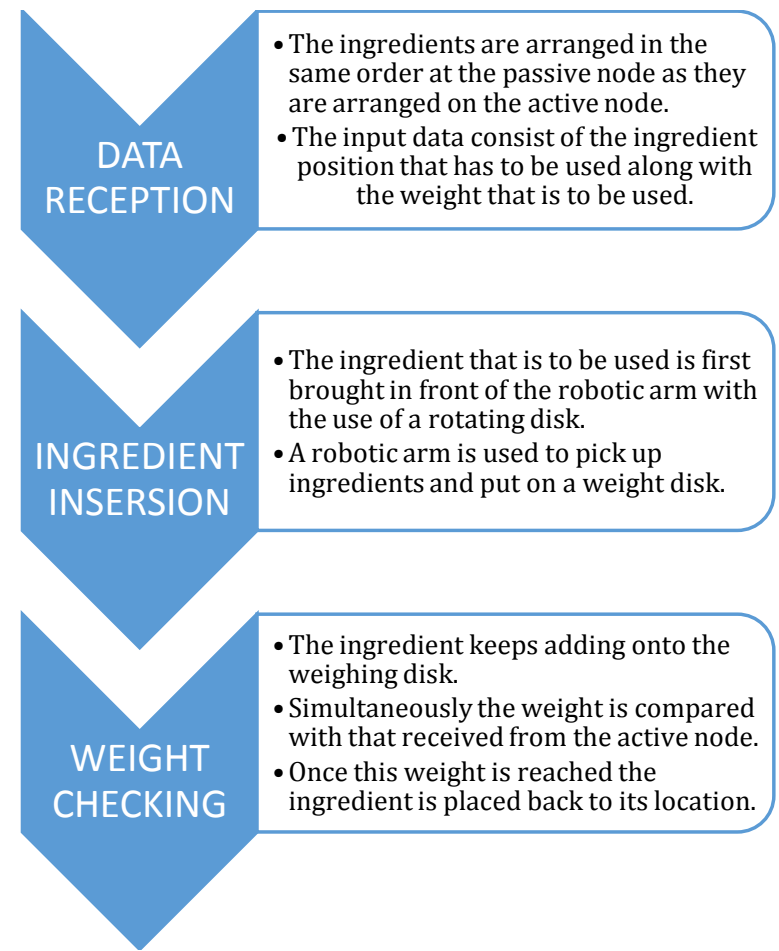

Fig -5 : Working of passive node

\section{List of Components Used}

- Motors.

- Sensors.

- Load cell

- RF Receiver.

- Atmega 328 microcontroller.

- HX 711 module

- Induction Cooker.

- $\quad$ Real Time Clock (RTC).
Table -2: List of sensors for passive node

\begin{tabular}{|l|l|}
\hline List of Sensors & $\begin{array}{l}\text { Functional Description in } \\
\text { brief }\end{array}$ \\
\hline Load cell & $\begin{array}{l}\text { Used to measure the weight } \\
\text { of the ingredient used. }\end{array}$ \\
\hline $\begin{array}{l}\text { Resistor } \\
\text { Device (RTD). Temperature }\end{array}$ & $\begin{array}{l}\text { To measure the temperature } \\
\text { of the container. }\end{array}$ \\
\hline
\end{tabular}

In practical, passive node receives the data from active node which is send to Atmega 328 to perform actuation of the motors of automatic cooking machine according to the given data synchronously. RF receiver performs the task of receiving the data.

Microcontroller gets the data from RF receiver and gives the control to the actuators to pick up the intended ingredient's container. After that we have to measure the proper amount of ingredient and insert in the pan. Then on the basis of received time parameter second ingredient is inserted and then this task gets repeated unless and until all the parameter have check. We also have to check the stirring parameter to stir the recipe and temperature parameter to heat the container to appropriate level. Here, we are using the servo motors in the automatic cooking machine. All the devices are interfaced to Atmega 328 on the Arduino board.

\subsection{The Microcontroller - Arduino Atmega 328}

The microcontroller we decided to build this module was the Arduino ATMega 328 [5], after careful reviews of several models. Considering that this single part is the heart of the module we selected with special care based on the characteristics like connectivity, available I/O. The microcontroller offered all the features we needed to develop the module.

ATMega 328 has a flash memory of $32 \mathrm{~KB}$, SRAM of 2 $\mathrm{KB}$, EPROM of $1 \mathrm{~KB}$ and clock speed of $20 \mathrm{MHz}$ The Arduino can be powered through the USB connections or with an external power supply

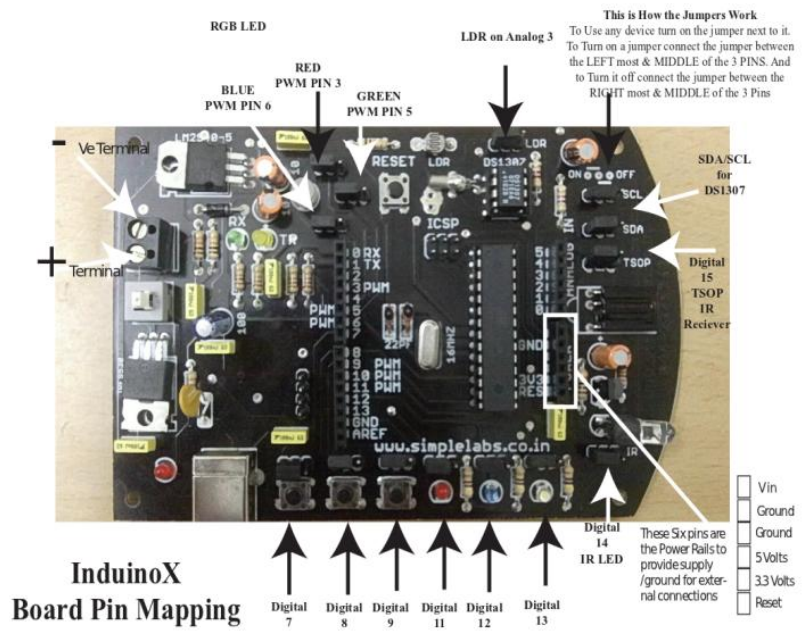

Fig -6: Arduino Board 
Additionally the Arduino platform was particularly attractive because of its open source physical computing platform based on a simple I/O board and the development environment that implements the Processing/Wiring language.

ATMega 328 has two 8 bit and one 16 bit timer/counter, real time counter with separable oscillator, 6 PWM channels, and execution of the instruction per clock cycle. In order to maximize the parallelism, the AVR uses Harvard Architecture - with separate memories and buses for program and data.

\subsection{HX 711 MODULE (ADC) 24-bit}

This 24-bit analog to digital and signal conditioning module is designed specifically for weight scales, weight sensor and industrial control applications to interface directly with a bridge sensor. The module is based on HX711 chip [6]. In most Load cells and weight sensors the output range of a strain gauge is very small and thus the signal needs to be amplified before processing to prevent introduction of errors. This module is used for amplifying the weight sensor and converting its analog sensor to digital one, therefore increasing the measurement accuracy. The output to micro-controller is serial. The module can be connected to 2 weight sensors at the same time. The module also provides power directly to sensors through out+ pin.

\section{Features:}

- $\quad$ Operating voltage range: $4.7 \sim 5.5 \mathrm{~V}$

- Operating temperature range: $-20 \sim+85$

- Can be used for 2 weight sensors at same time

- $\quad$ Fast and accurate 24-bit ADC

- Come with Faraday cage for noise reduction

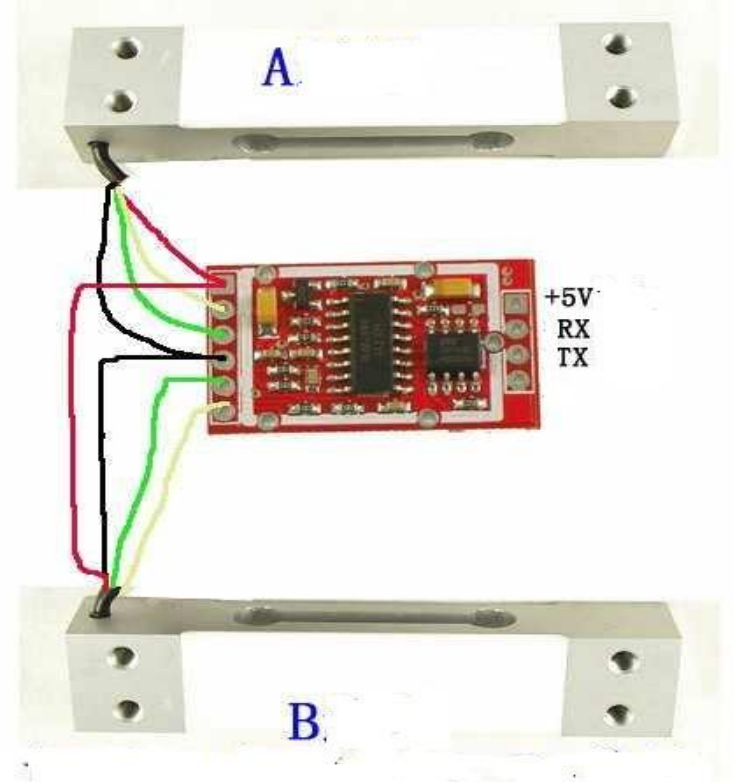

Fig -7: Interfacing of HX 711 Module with load cell
Table -3: Pin connection on the weight sensor side

\begin{tabular}{|l|l|l|}
\hline Function & Name & Color of wire \\
\hline Sensor power & Out+ & Red \\
\hline Signal -ve & A- & White \\
\hline Signal +ve & A+ & Green \\
\hline $\begin{array}{l}\text { Weight sensor } \\
\text { ground }\end{array}$ & GND & Black \\
\hline
\end{tabular}

Table -4: Pin connection on the arduino side

\begin{tabular}{|l|l|}
\hline Function & Name \\
\hline 5 V DC & VCC \\
\hline Digital Output & DO \\
\hline Clock & CK \\
\hline Ground & GND \\
\hline
\end{tabular}

\subsection{RF Module}

The RF module [7], as the name suggests, operates at Radio Frequency. The corresponding frequency range varies between $30 \mathrm{kHz} \& 300 \mathrm{GHz}$. In this RF system, the digital data is represented as variations in the amplitude of carrier wave. This kind of modulation is known as Amplitude Shift Keying (ASK) [3].

Transmission through RF is better than IR (infrared) because of many reasons. Firstly, signals through RF can travel through larger distances making it suitable for long range applications. Also, while IR mostly operates in lineof-sight mode, RF signals can travel even when there is an obstruction between transmitter \& receiver. Next, RF transmission is more strong and reliable than IR transmission. RF communication uses a specific frequency unlike IR signals which are affected by other IR emitting sources.

This RF module comprises of an RF Transmitter and an RF Receiver. The transmitter/receiver $(\mathrm{Tx} / \mathrm{Rx})$ pair operates at a frequency of 434 MHz. An RF transmitter receives serial data and transmits it wirelessly through RF through its antenna connected at pin4. The transmission occurs at the rate of $1 \mathrm{Kbps}-10 \mathrm{Kbps}$. The transmitted data is received by an RF receiver operating at the same frequency as that of the transmitter.

The RF module is often used along with a pair of encoder/decoder. The encoder is used for encoding parallel data for transmission feed while reception is decoded by a decoder. HT12E-HT12D, HT640-HT648, etc. are some commonly used encoder/decoder pair ICs. 


\section{Pin Connection:}
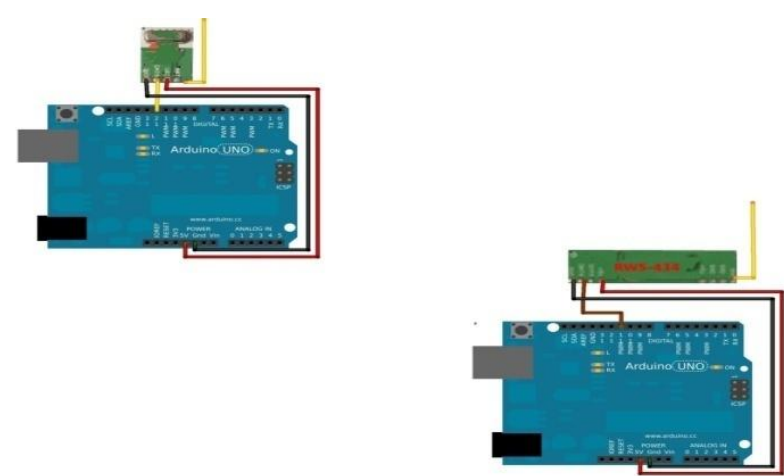

Fig -8: Interfacing of RF module with Arduino

Table- 5: Pin Description of RF Transmitter:

\begin{tabular}{|l|l|l|}
\hline $\begin{array}{l}\text { Pin } \\
\text { No }\end{array}$ & Function & Name \\
\hline 1 & Ground (0V) & Ground \\
\hline 2 & Serial data input pin & Data \\
\hline 3 & Supply voltage; 5V & VCC \\
\hline 4 & Antenna output pin & ANT \\
\hline
\end{tabular}

Table- 6: Pin Description of RF Receiver:

\begin{tabular}{|l|l|l|}
\hline $\begin{array}{l}\text { Pin } \\
\text { No }\end{array}$ & Function & Name \\
\hline 1 & Ground (0V) & Ground \\
\hline 2 & Serial data output pin & Data \\
\hline 3 & Linear output pin; not connected & NC \\
\hline 4 & Supply voltage; 5V & VCC \\
\hline 5 & Supply voltage; 5V & VCC \\
\hline 6 & Ground (0V) & Ground \\
\hline 7 & Ground (0V) & Ground \\
\hline 8 & Antenna input pin & ANT \\
\hline
\end{tabular}

\section{RESULTS}

As to successfully accomplish the task we need to be precise about the data collected and transmitted. So we calibrate the active node with the help of an external digital weighing machine and calculate the error in the readings of the device and weighing machine. Second thing to consider that to check if the transmitted data and the received data are same and that no error occurs while transmitting or receiving. We were able receive the correct data every time at the Passive Node.

\section{CONCLUSIONS}

The given module can further be improved in a number of ways. The module can be designed to compensate more ingredients along with an ingredient identification facility such as a barcode scanner, image processing [6]. In the current version we are using switches to identify ingredients. Even if simple this method lacks the freedom of placing the ingredients wherever one wishes to on the cooking module. If the ingredients are placed in wrong order it can prove disastrous. We therefore recommend use of a Barcode Scanner to identify ingredients this will eliminate the need of ingredients to be placed in the same order. Internet can be used as an interface instead of the RF module. The RF Module has a limited range and was used here as a temporary medium to transfer data. For the final product we recommend use of an Ethernet Shield to each and every Passive Node. Refrigeration facility can be provided to keep the ingredient at a particular temperature. In the current version the Active Node and the Passive Node are not reversible.

The project has two basic applications which are:

1. Preparation of food at a Remote Location.

2. Easy way to digitalize the recipe.

We are hoping that the current project can be manufactured as a product that will allow people to eat food prepared by a person of his choice. For example the person of choice can be a renowned chef. The chef may charge the person for this service. Also a smaller, more portable version will enable it to be taken wherever one wishes it to.

\section{REFERENCES}

[1]. H. Cho, "Dressing and modeling food," ACM SIGGRAPH 2007 courses. Course 6: Anyone can cook: inside Ratatouille's kitchen, pp. 7-21, 2007.

[2]. B.P.Lathi, "Modern Digital and Analog Communication Systems", Oxford University Press, 1998

[3]. Kanda poha, contributed by Khana Khajana, Sanjeev Kapoor, Available :http://www.sanjeevkapoor.com/kandapoha.aspx

[4]. Arduino, Available: http://www.arduino.cc

[5]. Hx711 module datasheet Available: http://www.dfrobot.com

[6]. S.R. Patil, Raveena Chavan, "Intelligent Cooking Providing Automatic Time and Temperature Setting Using Image Processing With Wide Range of Recipes for Microwave Ovens", Department of Computer Engineering, Pune University, Pune, India. received on December, 2013.

[7]. Documentation for the VirtualWire 1.3, communications library for Arduino by Mike McCauley

\section{BIOGRAPHIES}

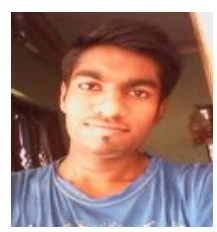

Rajesh Asutkar, Department of Electronics and Telecommunication, SGGSIE\&T, Nanded- 431606, India,

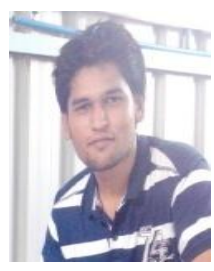

Gaurav Satav,Department of Electronics and Telecommunication,SGGSIE\&T, Nanded- 431606, India, 